Research Article

\title{
New One-Sided EWMA $t$ Charts without and with Variable Sampling Intervals for Monitoring the Process Mean
}

\author{
Yan Wang $\mathbb{D}^{1},{ }^{1}$ Xuelong Hu $\mathbb{D}^{1},{ }^{1}$ Xiaojian Zhou $\mathbb{D}^{1},{ }^{1}$ Yulong Qiao $\mathbb{D}^{2},{ }^{2}$ and Shu Wu $\mathbb{D}^{3}$ \\ ${ }^{1}$ School of Management, Nanjing University of Posts and Telecommunications, Nanjing 210003, China \\ ${ }^{2}$ School of Automation, Nanjing University of Science and Technology, Nanjing 210094, China \\ ${ }^{3}$ School of Logistics, Wuhan University of Technology, Wuhan, China \\ Correspondence should be addressed to Xuelong Hu; hxl0419@njupt.edu.cn
}

Received 21 August 2020; Revised 27 October 2020; Accepted 3 November 2020; Published 26 November 2020

Academic Editor: Mahmoud Mesbah

Copyright (c) 2020 Yan Wang et al. This is an open access article distributed under the Creative Commons Attribution License, which permits unrestricted use, distribution, and reproduction in any medium, provided the original work is properly cited.

In statistical process control (SPC), $t$ charts play a vital role in the monitoring of the process mean, especially when the process variance is unknown. In this paper, two separate upper-sided and lower-sided exponentially weighted moving average (EWMA) $t$ charts are first proposed and the Monte Carlo simulation method is used to obtain their run length (RL) properties. Compared with the traditional one-sided EWMA $t$ charts and several run rules $t$ charts, the proposed charts are proven to have better performance than these competing charts. In addition, by adding the variable sampling interval (VSI) feature to the proposed charts, the new VSI one-sided EWMA $t$ charts are shown to detect different shift sizes in the process more efficient than the chart without VSI feature. Finally, an example of a milk filling process illustrates the use of the charts.

\section{Introduction}

In a production process, the quality of a product is usually affected by some chance or assignable causes. The chancecaused variation is unavoidable and inevitably occurs in a process. While variation by assignable causes means that there exist unwanted factors to be detected. A process is said to be statistically in-control if only a chance-caused variation exists in a process. Otherwise, the process is deemed to be out-of-control.

As an important tool of statistical process control (SPC), the $\bar{X}$ chart has been widely used to decide whether the production process is in-control or out-of-control (see Maragah and Woodall [1], Chan and Cui [2], Chen and Cheng [3], and Ho and Trindade [4], to name a few). However, as pointed by Zhang et al. [5], the $\bar{X}$ chart was not robust against estimation errors or changes in process standard deviation and instead, they suggested $t$ and exponentially weighted moving average (EWMA) $t$ charts for the process monitoring. Following this work, Tran [6] proposed several run rules $t$ control charts to monitor process mean changes. Sitt et al. [7] investigated the properties of the run sum $t$ chart based on the idea of integrating the rum sum chart with a $t$ chart. Castagliola et al. [8] developed the variable sample size $t$ control chart for monitoring short production runs. All the above research works showed that $t$ charts are more robust than $\bar{X}$ charts when the process standard deviation is unknown. In addition, it was also known that the EWMA $t$, cumulative sum (CUSUM) $t$, and Shewhart $t$ charts have different sensitivities for different shifts. For small to moderate shifts, the EWMA $t$ and CUSUM $t$ charts perform better than the Shewhart $t$ chart. While for large shifts, the Shewhart $t$ chart performs better than other two types of charts $[9,10]$.

In recent years, EWMA $t$ charts have received much attention due to its ability to detect small shifts quickly. For example, Zhang et al. [5] first discussed the properties of the $t$ and EWMA $t$ charts. Then, Celano et al. [11] studied the design of the EWMA $t$ chart based on the triangle distribution and the uniform distribution. Calzada and Scariano [12] presented the synthetic $t$ control chart and the synthetic EWMA $t$ control chart with optimal control limits. To deal with the problem that the conventional EWMA $t$ control chart was only sensitive to a fixed shift, Chang and Sun [13] 
proposed an adaptive EWMA $t$ control chart, and it was shown that the adaptive EWMA $t$ control chart has similar average run length (ARL) performance with the EWMA $t$ chart but with a smaller standard deviation of run length (SDRL) value. In addition, to improve the performance of the fixed sampling interval (FSI) EWMA $t$ chart, the EWMA $t$ chart with variable sampling intervals (VSIs) was proposed by Kazemzadeh et al. [14].

Through the review of the above research works, it is found that all control charts mentioned were constructed on two-sided EWMA $t$ charts. However, in fact, two-sided EWMA charts do have the inertia problem. If the EWMA statistic happens to be below its center line, and at the same time, an upper-sided shift occurs, then the EWMA chart needs more samples to detect the shift and vice versa. To overcome the inertia problem, some researchers suggested two separate one-sided EWMA control charts with a "restart state." For example, Castagliola et al. [15] proposed two onesided EWMA charts to monitor the coefficient of variation (CV) and suggested the optimal parameters. Tran et al. [16] presented two one-sided EWMA charts for monitoring the ratio of two normal variables and calculated the optimal smoothing coefficient, control limits, and ARL values of the chart. The results showed that the one-sided EWMA control charts performed effectively than two-sided EWMA charts when the mean shift direction of the process is known prior. $\mathrm{Hu}$ et al. [17] investigated a one-sided EWMA $t$ chart with a traditional reset model to monitor the process mean. All the research works showed that one-sided EWMA $t$ charts are superior to two-sided EWMA $t$ charts, especially for small or moderate shifts.

Motivated by the above research works, this paper proposes an improved one-sided EWMA $t$ chart with a new reset model, which was recently suggested by Zhang et al. [18] for monitoring the CV in a process. It is shown that the newly developed scheme performs better than some existing charts. Moreover, in order to further improve the sensitivity of the chart, we also study the performance of the proposed one-sided EWMA $t$ charts with variable sampling interval (VSI) feature.

The remainder of this paper is organized as follows. In Section 2, the traditional one-sided EWMA $t$ charts are reviewed. New one-sided EWMA $t$ charts are then introduced in Section 3. In Section 4, the Monte Carlo simulation method is used to obtain the ARL, MRL (median run length), and SDRL performances for some selected values of the design parameters. Section 5 presents the detailed construction of the new one-sided EWMA $t$ chart with VSI feature and presents the out-of-control ATS (average time to signal) profiles of the VSI chart for different combinations of sample size and smoothing parameter. Following this, an illustrative example is presented to show the implementation of the new chart in Section 6. In Section 7, some conclusions and recommendations are presented.

\section{Traditional One-Sided EWMA $t$ Charts}

2.1. The $t$ Statistic. Suppose that a set of data $\left\{X_{i, 1}, X_{i, 2}, X_{i, 3}\right.$, $\left.\ldots, X_{i, j}\right\}$ is observed at time $i$, where $j$ represents the number of item in the $i^{\text {th }}$ sample. In addition, it is assumed that $X_{i, j} \sim N\left(\mu_{0}+\delta \sigma_{0}, \sigma_{0}\right)$, where $\mu_{0}$ and $\sigma_{0}$ are the in-control process mean and standard deviation, respectively, and $\delta$ is the parameter of the process mean shift. The sample mean and standard deviation are given as follows:

$$
\begin{aligned}
\bar{X}_{i} & =\frac{1}{n} \sum_{j=1}^{n} X_{i, j}, \\
S_{i} & =\sqrt{\frac{1}{n-1} \sum_{j=1}^{n}}\left(X_{i, j}-\bar{X}_{i}\right)^{2} .
\end{aligned}
$$

The statistic $T_{i}$ is equal to

$$
T_{i}=\frac{\bar{X}_{i}-\mu_{0}}{S_{i} / \sqrt{n}} .
$$

When the process is in-control $(\delta=0)$, the $T_{i}$ in equation (2) follows a student $t$ distribution with cumulative distribution function (c.d.f.) $F_{t}(\cdot \mid n-1)$ with $n-1$ degrees of freedom. When the process is out-of-control $(\delta \neq 0)$, the statistic $T_{i}$ can be simplified to

$$
\begin{aligned}
T_{i} & =\frac{\bar{X}_{i}-\mu_{1}+\mu_{1}-\mu_{0}}{S_{i} / \sqrt{n}} \\
& =\frac{\sqrt{n}\left(\bar{X}_{i}-\mu_{1}\right) / \sigma_{0}+\sqrt{n}\left(\mu_{1}-\mu_{0}\right) / \sigma_{0}}{S_{i} / \sigma_{0}} \\
& =\frac{Z_{i}+\delta \sqrt{n}}{\sqrt{V_{i} / n-1}},
\end{aligned}
$$

where $Z_{i}=\sqrt{n}\left(\bar{X}_{i}-\mu_{1}\right) / \sigma_{0}$ follows a standard normal distribution and $V_{i}=(n-1) S_{i}^{2} / \sigma_{0}^{2}$ follows a chi-square distribution. The statistic $T_{i}$ in equation (3) can be easily proved to follow a noncentral $t$ distribution $F_{t}(\cdot \mid n-1, \varphi)$ with $n-1$ degrees of freedom and noncentral parameter $\varphi=\delta \sqrt{n}$.

2.2. A Brief Review of Traditional One-Sided EWMA t Charts. In this section, we give a brief review of traditional one-sided EWMA $t$ charts. First, when $\delta>0$, the traditional uppersided EWMA (U-EWMA) $t$ chart is defined as

$$
Y_{i}^{+}=\max \left(t_{0},(1-\lambda) Y_{i-1}^{+}+\lambda T_{i}\right),
$$

with $Y_{i}^{+}=t_{0}=0$ as the initial value and the upper control limit $\mathrm{UCL}^{+}$. Second, when $\delta<0$, the traditional lower-sided EWMA (L-EWMA) $t$ chart is defined as

$$
Y_{i}^{-}=\min \left(t_{0},(1-\lambda) Y_{i-1}^{-}+\lambda T_{i}\right),
$$

with $Y_{i}^{-}=t_{0}=0$ as the initial value and the lower control limit $\mathrm{LCL}^{-}$. The parameter $\lambda \in(0,1]$ is the smooth constant of the two separate one-sided EWMA $t$ charts. However, it can be noted from equation (4) that when $t_{0}>$ $(1-\lambda) Y_{i-1}^{+}+\lambda T_{i}$, then $Y_{i}^{+}=t_{0}$ and $Y_{i+1}^{+}=\max \left(t_{0}\right.$, $\left.(1-\lambda) t_{0}+\lambda T_{i}\right)$. Obviously, the samples information collected before time $i+1$ are not used any more. As a result, it violates the characteristics of EWMA type control charts that 
use the information of both past and current samples. To solve this problem, new one-sided EWMA $t$ charts are proposed and the detailed results are described in the next section.

\section{New One-Sided EWMA $t$ Control Charts}

To use the information of the current and past samples and improve the sensitivity of the traditional one-sided EWMA $t$ charts, the new upper-sided EWMA (denoted as U-NEWMA) $t$ is defined as

$$
Y_{i}^{+}=\max \left(t_{0}, \lambda T_{i}+(1-\lambda) Y_{i-1}\right),
$$

where $Y_{i}=\lambda T_{i}+(1-\lambda) Y_{i-1}$ and $Y_{i}^{+}=t_{0}=0$ is the initial value and the upper control limit is $\mathrm{UCL}^{+}$. If $Y_{i}^{+}>\mathrm{UCL}^{+}$, an out-of-control signal is triggered. Second, when $\delta<0$, the new lower-sided EWMA (denoted as L-NEWMA) $t$ is defined as

$$
Y_{i}^{-}=\min \left(t_{0}, \lambda T_{i}+(1-\lambda) Y_{i-1}\right),
$$

with $Y_{i}^{-}=t_{0}=0$ as the initial value and the upper control limit is $\mathrm{LCL}^{-}$. If $Y_{i}^{-}<\mathrm{LCL}^{-}$, an out-of-control signal is triggered. It is expected that new one-sided EWMA $t$ charts constructed using all the samples information are more effective than the traditional one-sided EWMA $t$ charts. It is noted that if the direction of the shift is not known in advance, the new upper-sided and lower-sided EWMA $t$ charts can be used in combination. Therefore, the practitioners can allocate the type I error between two separate one-sided EWMA $t$ charts to guarantee the desired $\mathrm{ARL}_{0}$ of the combined new EWMA $t$ charts, and the combined charts give a signal if $Y_{i}^{+}>\mathrm{UCL}^{+}$or $Y_{i}^{-}<\mathrm{LCL}^{-}$.

\section{Numerical Results and Comparisons}

The properties of the new one-sided EWMA $t$ charts, simulated using Monte Carlo procedure, are presented in this section. Several measures, such as ARL, SDRL, and MRL, are used to evaluate the performance of the charts. The ARL is the expect number of samples from the beginning of the monitoring to the appearance of an out-of-control signal. The MRL is the median value of the run length (RL) values, and the SDRL is the standard deviation of the RL values. When the process is in-control, the ARL is denoted as $\mathrm{ARL}_{0}$. When the process is out-of-control, the ARL is denoted as $A R L_{1}$. In the related studies of control charts, it is necessary to ensure the same $\mathrm{ARL}_{0}$ for all charts, and at the same time, the smaller the value of $\mathrm{ARL}_{1}$, the better the performance of a control chart. In addition, the smaller the value of SDRL, the better the performance of a control chart, and the RL distribution of the control chart is less dispersed than the one with a larger SDRL value.

In general, three methods, i.e., integral equation, Markov chain, and Monte Carlo simulations, were usually used for calculating the RL properties of a control chart. In this paper, we use the Monte Carlo simulation method to compute the properties of the control chart due to its simplicity. Moreover, a bisection search algorithm is used to find the
$\mathrm{UCL}^{+}$and $\mathrm{LCL}^{-}$of the new one-sided EWMA $t$ charts for a specified value of $\lambda$. Some parameter combinations $(\lambda$, $\left.\mathrm{UCL}^{+}\right)$and $\left(\lambda, \mathrm{LCL}^{-}\right)$of the new one-sided EWMA $t$ charts are presented in Tables 1 and 2 when the desired $\mathrm{ARL}_{0}=200, n \in\{5,7,9,11\}$, and $\lambda \in\{0.1,0.2,0.3,0.4,0.5$, $0.75,0.9,1.0\}$.

Since the $t$ distribution is symmetric, we can note that the $\mathrm{LCL}^{-}$values in Table 2 are the opposite values of the $\mathrm{UCL}^{+}$in Table 1. It can be concluded that the $\mathrm{UCL}^{+}$of the U-NEWMA $t$ charts increases when $\lambda$ increases or $n$ decreases, and the $\mathrm{LCL}^{-}$of the L-NEWMA $t$ charts decreases when $\lambda$ increases or $n$ decreases. For example, when $n=5$ (see Table 1), the $\mathrm{UCL}^{+}$increases from 0.6811 up to 4.5961 when $\lambda$ increases from 0.1 up to 1 . When $\lambda=0.1, \mathrm{UCL}^{+}$ decreases from 0.6811 down to 0.5430 when $n$ increases from 5 up to 11. Similarly, in Table 2 , when $n=5$, the $\mathrm{LCL}^{-}$ decreases from -0.6811 down to -4.5961 when $\lambda$ increases from 0.1 up to 1 . When $\lambda=0.1$, the $\mathrm{LCL}^{-}$increases from -0.6811 up to -0.5430 when $n$ increases from 5 up to 11 .

4.1. Comparisons with Traditional One-Sided EWMA tCharts. In this section, the out-of-control (ARL, MRL, SDRL) performances of the proposed chart are compared with the ones of the traditional one-sided EWMA $t$ charts when $\mathrm{ARL}_{0}=200, \lambda \in\{0.1,0.2,0.3,0.4,0.5,0.75,0.9\}, \delta \in\{0,0.1$, $0.3,0.5,0.7,1,1.5,2,3\}$, and $n \in\{5,7,9,11\}$ (see Tables 3 and 4). Here, we only present the performances of the uppersided charts for different shifts. Similarly, the performances of the lower-sided charts can also be obtained. From Tables 3 and 4 , it can be concluded that the proposed upper-sided NEWMA $t$ chart has smaller ARL, MRL, and SDRL values compared with the ones of the traditional upper-sided EWMA $t$ chart. For example, when $\lambda=0.1, n=5$, and $\delta \in\{0.1$, $0.3,0.5,0.7,1,1.5,2,3\}$, the corresponding ARLs of the U-NEWMA $t$ chart are $\{56.71,14.41,7.45,5.04,3.46,2.38$, $1.90,1.43\}$, respectively. While for the U-EWMA $t$ chart, the corresponding ARLs are $\{68.82,17.10,8.59,5.72,3.88,2.64$, $2.07,1.56\}$, which are all larger than the ones of the proposed chart. Also, when $\lambda=0.1, n=5$, and $\delta \in\{0.1,0.3,0.5,0.7,1$, $1.5,2,3\}$, the MRL values of U-EWMA $t$ are $\{50,14,8,5,4,3$, $2,2\}$ and the SDRL values are $\{62.30,11.24,4.23,2.36,1.38$, $0.85,0.65,0.51\}$. While for the U-NEWMA $t$ chart, the corresponding MRLs are $\{41,12,7,5,3,2,2,1\}$ and the corresponding SDRLs are $\{51.02,9.35,3.69,2.11,1.26,0.78$, $0.60,0.50\}$.

From Table 3, it can also be noted that a small value of $\lambda$ is more sensitive to small shifts in the process. For example, the ARL of the U-NEWMA $t$ chart increases from 56.71 up to 126.47 as $\lambda$ increases from 0.1 up to 0.9 when $\delta=0.1$ and $n=5$. In addition, the ARL decreases with the increase in $\delta$. For example, as $\delta$ increases from 0.1 up to 3 , the ARL of the U-NEWMA $t$ chart decreases from 56.71 down to 1.43 when $\lambda=0.1$ and $n=5$. Moreover, the U-NEWMA $t$ chart performs much better than the U-EWMA $t$ chart for a small smoothing parameter $\lambda$. For a large value of $\lambda$, the difference between U-EWMA $t$ chart and U-NEWMA $t$ chart is negligible. For example, in Table 4, when $\delta=3$ and $n=11$, regardless of the value of $\lambda$, all the value of ARLs are 1 . 
TABLE 1: The $\mathrm{UCL}^{+}$of the U-NEWMA $t$ chart when $\mathrm{ARL}_{0}=200$.

\begin{tabular}{lcccc}
\hline$\lambda$ & $n=5$ & $n=7$ & $n=9$ & $n=11$ \\
\hline 0.10 & 0.6811 & 0.5958 & 0.5614 & 0.5430 \\
0.20 & 1.1493 & 0.9854 & 0.9227 & 0.8880 \\
0.30 & 1.5763 & 1.3314 & 1.2367 & 1.1838 \\
0.40 & 1.9932 & 1.6610 & 1.5315 & 1.4634 \\
0.50 & 2.4137 & 1.9866 & 1.8214 & 1.7343 \\
0.75 & 3.4872 & 2.8192 & 2.5577 & 2.4219 \\
0.90 & 4.1474 & 3.3416 & 3.0280 & 2.8575 \\
1.00 & 4.5961 & 3.7043 & 3.3526 & 3.1667 \\
\hline
\end{tabular}

TABLE 2: The $\mathrm{LCL}^{-}$of the L-NEWMA $t$ chart when $\mathrm{ARL}_{0}=200$.

\begin{tabular}{lcccc}
\hline$\lambda$ & $n=5$ & $n=7$ & $n=9$ & $n=11$ \\
\hline 0.10 & -0.6811 & -0.5958 & -0.5614 & -0.5430 \\
0.20 & -1.1493 & -0.9854 & -0.9227 & -0.8880 \\
0.30 & -1.5763 & -1.3314 & -1.2367 & -1.1838 \\
0.40 & -1.9932 & -1.6610 & -1.5315 & -1.4634 \\
0.50 & -2.4137 & -1.9866 & -1.8214 & -1.7343 \\
0.75 & -3.4872 & -2.8192 & -2.5577 & -2.4219 \\
0.90 & -4.1474 & -3.3416 & -3.0280 & -2.8575 \\
1.00 & -4.5961 & -3.7043 & -3.3526 & -3.1667 \\
\hline
\end{tabular}

Similar conclusions hold for the MRL and SDRL performances of these EWMA $t$ charts.

4.2. Comparisons with One-Sided Run Rules $t$ Charts. In practice, Shewhart type control charts with supplementary run rules also show strong sensitivity in detecting small or moderate process shifts. Some popular run rules were suggested by Divoky and Taylor [19], Bissell [20], Roberts [21], Champ and Woodall [22], Lowry et al. [23], Palm [24], Shmueli and Cohen [25], Zhang and Castagliola [26], and Tran [27].

Recently, Tran [6] proposed several new run rules $t$ control charts to monitor changes in the process mean. These run rules charts, which are based on the traditional Shewhart $t$ chart, were more efficient than the traditional $t$ charts. Here, we mainly choose three kinds of run rules $t$ charts as a comparison with the proposed one-sided EWMA $t$ charts. The run rules schemes are 2-out-of-3, 3-out-of-4, and 4-out-of-5, respectively. It is worth noting that the aforementioned run rules $t$ charts are all two sided. For fair comparisons, we adapt these run rules to the one-sided cases (see Amdouni et al. [28]). Considering the representability and simplicity of the $\mathrm{RR}_{2,3}-t$ control chart, we only present the details of the upper-sided 2 -of- 3 run rules $t$ chart. The corresponding transition probability matrix $Q$ is the $(7,7)$ matrix of transient probabilities

$$
Q=\left(\begin{array}{ccccccc}
0 & 0 & 0 & \text { PC } & \text { PL } & 0 & 0 \\
0 & 0 & 0 & 0 & 0 & 0 & \text { PC } \\
\text { PC } & \text { PL } & 0 & 0 & 0 & 0 & 0 \\
0 & 0 & \text { PU } & \text { PC } & \text { PL } & 0 & 0 \\
0 & 0 & 0 & 0 & 0 & \text { PU } & \text { PC } \\
\text { PC } & 0 & 0 & 0 & 0 & 0 & 0 \\
0 & 0 & \text { PU } & \text { PC } & 0 & 0 & 0
\end{array}\right) .
$$

The probabilities PL, PC, and PU are equal to

(i) For the upper-sided $\mathrm{RR}_{2,3}-t$ chart, $\mathrm{PU}=1-\mathrm{F}\left(\mathrm{UCL}^{+}\right)$, $\mathrm{PC}=\mathrm{F}\left(\mathrm{UCL}^{+}\right)$, and $\mathrm{PL}=0$

(ii) For the lower-sided $\mathrm{RR}_{2,3}-t$ chart, $\mathrm{PL}=\mathrm{F}\left(\mathrm{LCL}^{-}\right)$, $\mathrm{PC}=1-\mathrm{F}\left(\mathrm{LCL}^{-}\right)$, and $\mathrm{PU}=0$

where $\mathrm{UCL}^{+}\left(\mathrm{LCL}^{-}\right)$is the upper (lower) control limit of the $\mathrm{RR}_{2,3^{-}} t$ chart. For the $\mathrm{RR}_{3,4^{-}} t$ chart and $\mathrm{RR}_{4,5^{-}} t$ chart, the matrix $Q$ is not presented here and readers can refer to Tran [6] and Castagliola et al. [29] for more details.

The (ARL, MRL, SDRL) profiles of the U-NEWMA $t$ chart and several run rules $t$ charts are presented in Table 5 when $\mathrm{ARL}_{0}=200, n \in\{5,7,9,11\}, \delta \in\{0,0.1,0.3,0.5,0.7,1$, $1.5,2,3\}$, and $\lambda \in\{0.1,0.2,0.3,0.5,1\}$. For small values of $\delta$, the U-NEWMA $t$ chart with a small smoothing parameter $\lambda$ performs much better than the run rules $t$ control charts. For example, when $n=5$ and $\delta=0.1$, the corresponding ARL of the U-NEWMA $t$ chart with $\lambda=0.1$ is equal to 56.71 and the corresponding ARLs of three upper-sided run rules $t$ charts are $100.18,87.36$, and 80.80 , respectively. While for the detection of large shifts $\delta$, the performance of the U-NEWMA $t$ chart with a large $\lambda$ is better than that of the run rules $t$ charts. For example, when $n=5$ and $\delta=3$, the corresponding ARL of U-NEWMA $t$ chart with $\lambda=1$ is equal to 1.12 and the corresponding ARLs of three upper-sided run rules $t$ charts are 2.00,3.00, and 4.00, respectively. Moreover, it can be seen that the MRL and SDRL of the proposed chart perform similarly as the ARL.

\section{New One-Sided EWMA $t$ Control Charts with Variable Sampling Intervals}

5.1. Construction of VSI One-Sided NEWMA $t$ Charts. One-sided EWMA $t$ charts proposed in Section 3 adopt a fixed sampling interval (FSI) scheme, which ensures that the time interval between two successive samples is fixed. However, according to the work conducted in [14] on the traditional EWMA $t$ control chart, it is found that the VSI scheme is more sensitive than the FSI scheme in detecting process mean changes. Motivated by this, we integrate the VSI scheme into new one-sided EWMA $t$ charts in Section 3 (denoted as VSI one-sided NEWMA $t$ chart). Different from the FSI scheme, this scheme divides the control region of the chart into three parts: the central area, the warning area, and the out-of-control area. When the charting statistics in equation (6) or (7) fall in the central area, it indicates that the process is in a safe state and the next sample could be taken after a long sampling interval $h_{L}$. When the charting statistics in equation (6) or (7) fall in the warning area, it indicates that the process is facing the risk of an out-ofcontrol state. It is necessary to be careful, and the next sample should be taken after a short sampling interval $h_{S}$. Once the charting statistics fall into the out-of-control area, it can be concluded that the process is out-of-control.

As a pioneer in the study of VSI charts, Reynolds et al. [30] first studied the performance of $\bar{X}$ charts with VSI feature. By observing the signs of charting statistics, different sampling intervals can be flexibly selected. For more recent 


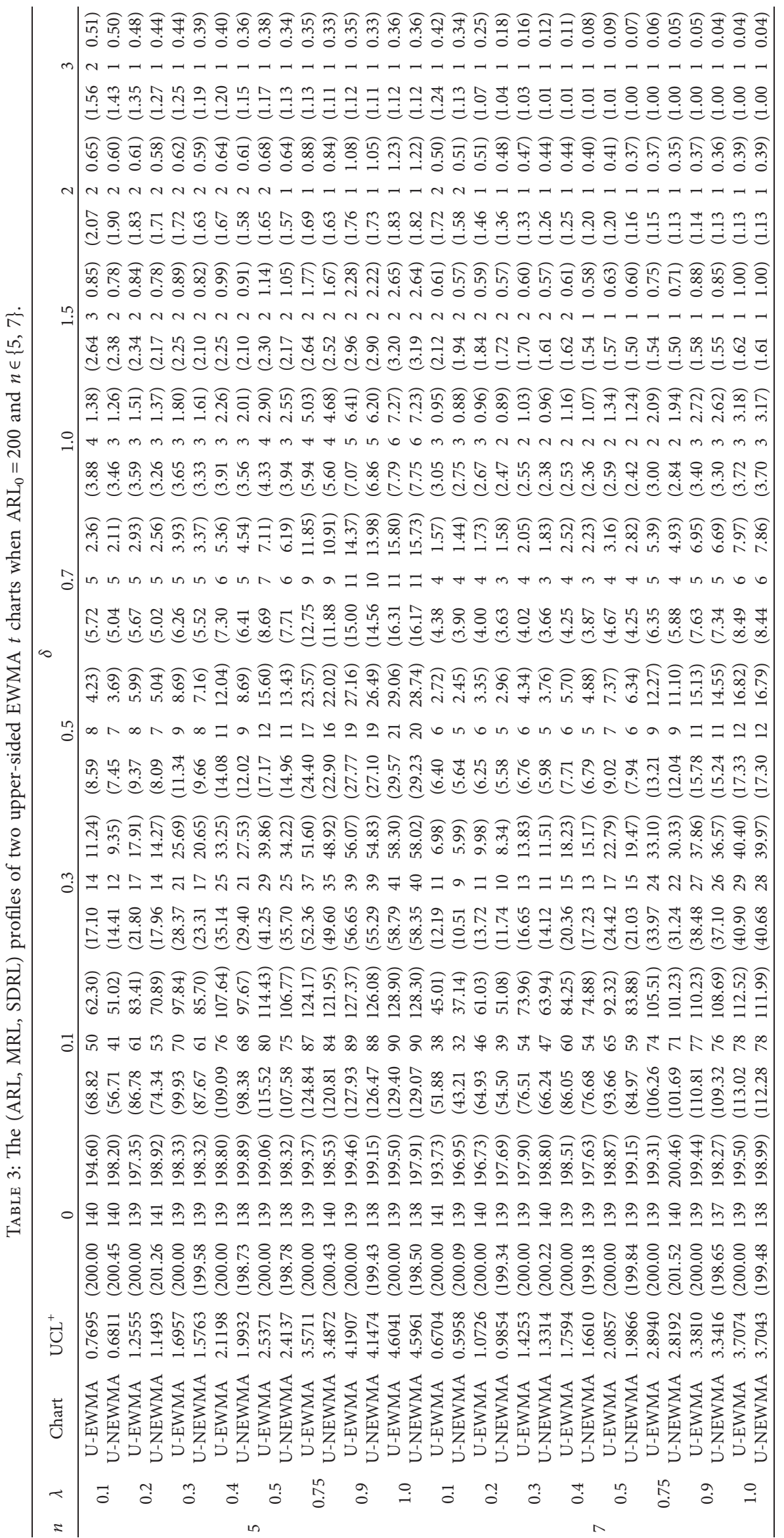




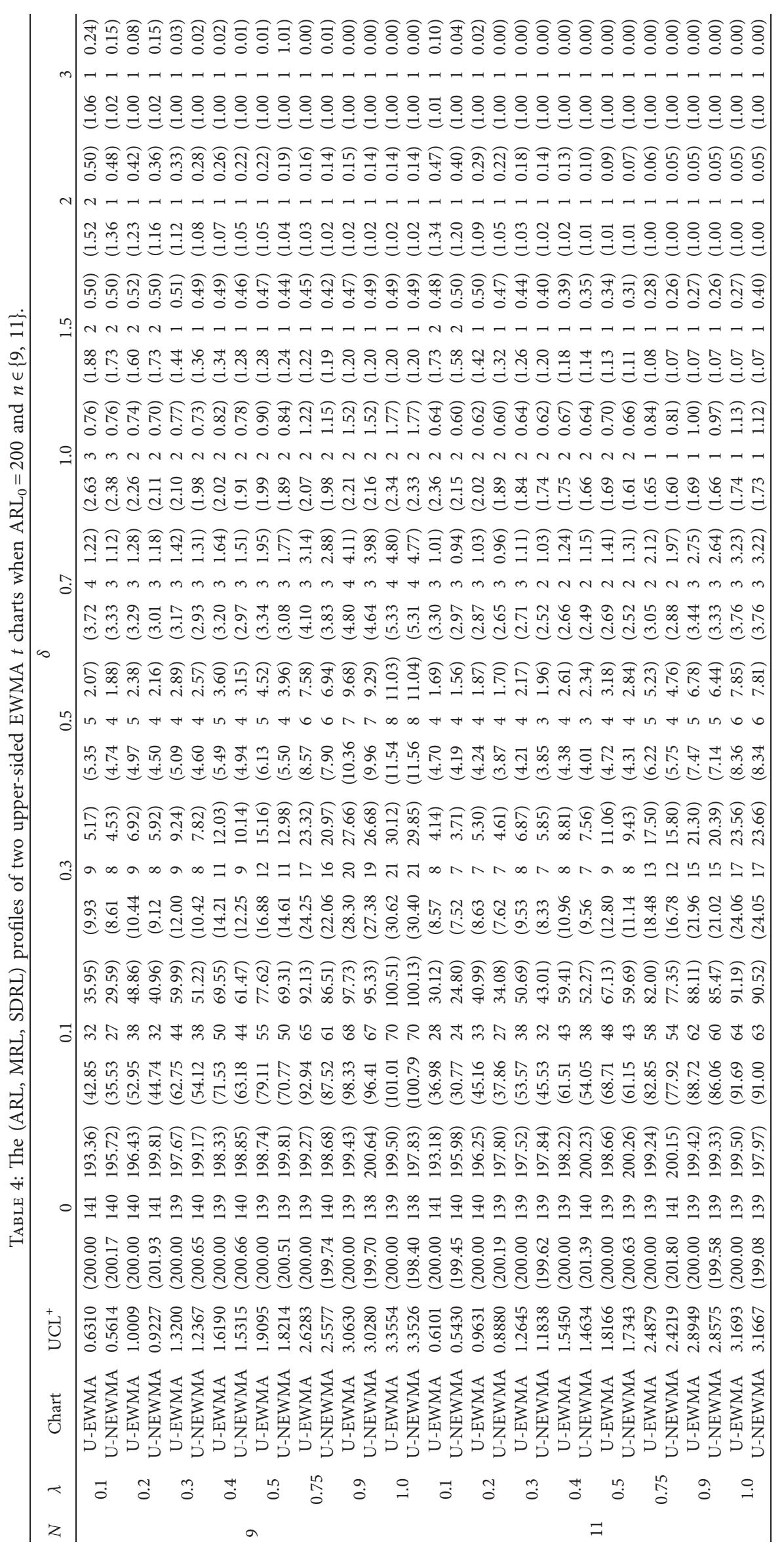




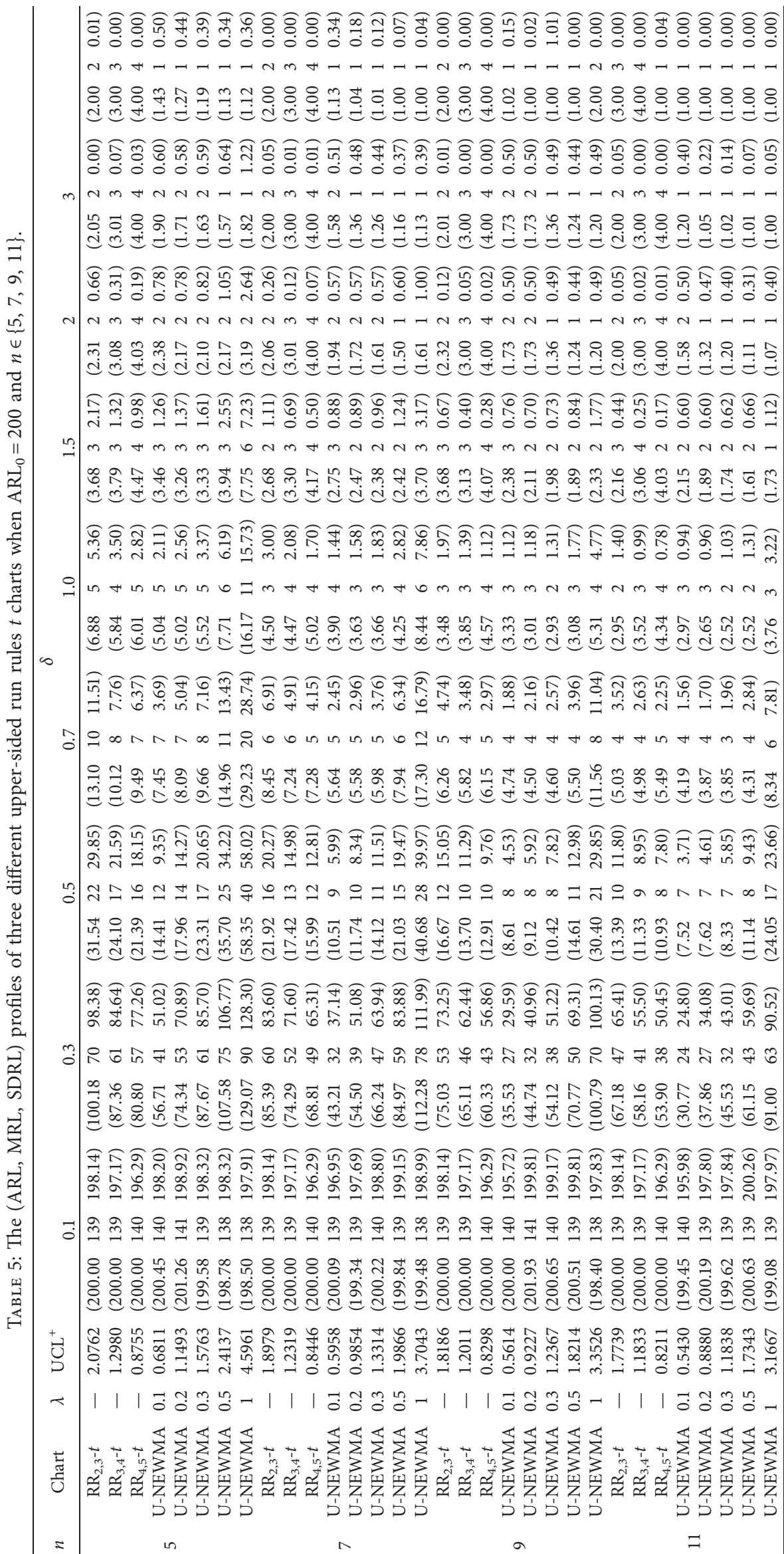


works on VSI control charts, readers may refer to Coelho et al. [31], Haq [32], and Nguyen et al. [33] to name a few. These research works showed that VSI control charts were superior to the corresponding FSI charts. Inspired by these works, we propose VSI one-sided NEWMA $t$ charts to monitor the process mean in this section.

For VSI one-sided NEWMA $t$ charts, the control limits $\mathrm{UCL}^{+}$and $\mathrm{LCL}^{-}$are the same as those in the original onesided FSI charts. In addition, $\mathrm{UWL}^{+}$and $\mathrm{LWL}^{-}$are the upper and lower warning limits of the upper-sided and lower-sided NEWMA $t$ charts, respectively. For the upper-sided VSI NEWMA $t$ chart, a short sampling interval $h_{S}$ is used when the current statistic $Y_{i}^{+}$in equation (6) falls within the region $\left[0, \mathrm{UWL}^{+}\right]$and a long sampling interval $h_{L}$ is used when the current statistic $Y_{i}^{+}$falls within the region $\left[\mathrm{UWL}^{+}, \mathrm{UCL}^{+}\right]$. Similarly, for the lower-sided VSI NEWMA $t$ chart, a short sampling interval $h_{S}$ is used when the current statistic $Y_{i}^{-}$in equation (7) falls within the region $\left[\mathrm{LWL}^{-}, 0\right]$ and a long sampling interval $h_{L}$ is used when the current statistic $Y_{i}^{-}$ falls within the region $\left[\mathrm{LCL}^{-}, \mathrm{LWL}^{-}\right]$. At the beginning of process monitoring where there is no sample information before, a short sampling interval $h_{S}$ is suggested to avoid the potential problems in the start-up of process.

When the process is in-control, the values of $h_{S}$ and $h_{L}$ are determined by satisfying the following equations:

$$
\left\{\begin{array}{l}
\rho_{1} h_{S}+\rho_{2} h_{L}=1, \\
\rho_{1}+\rho_{2}=1,
\end{array}\right.
$$

where $\rho_{1}$ and $\rho_{2}$ are the long run proportions of sampling intervals $h_{S}$ and $h_{L}$, respectively. Moreover, through a lot of simulations, we found that the value of $\rho_{1}$ is always smaller than 0.5 and the value of $\rho_{2}$ is always larger than 0.5 . In this paper, $\rho_{1}=0.4, \rho_{2}=0.6$, and $\left(h_{S}, h_{L}\right)=(0.1,1.6)$ are selected for discussion.

5.2. Comparison with FSI One-Sided NEWMA $t$ Charts. For the VSI NEWMA $t$ control chart, since the sampling intervals between two successive samples varied, the ATS was generally used instead of ARL to evaluate the performance of different charts. ATS is defined as the expected time from the beginning of the monitoring to an out-ofcontrol signal. Tables 6 and 7 present the out-of-control ATS (denoted as $\mathrm{ATS}_{1}$ ) profiles of the VSI and FSI upper-sided NEWMA $t$ charts when $\lambda \in\{0.1,0.2,0.3,0.4,0.5,0.75,0.9\}$, $\delta \in\{0,0.1,0.3,0.5,0.7,1,1.5,2,3\}$, and $n \in\{5,7,9,11\}$. It can be concluded that the proposed VSI upper-sided NEWMA $t$ chart has smaller ATS $_{1}$ values compared with the FSI uppersided NEWMA $t$ chart. For example, when $\lambda=0.1, n=5$, and $\delta \in\{0.1,0.3,0.5,0.7,1,1.5,2,3\}$, the corresponding $\mathrm{ATS}_{1}$ values of the VSI upper-sided NEWMA $t$ chart are $\{33.54$, $3.97,1.44,0.76,0.40,0.24,0.19,0.14\}$, respectively. While for the FSI upper-sided NEWMA $t$ chart, the corresponding $\mathrm{ATS}_{1}$ values are $\{56.71,14.41,7.45,5.04,3.46,2.38,1.90$, $1.43\}$, which are all larger than the ones of the proposed VSI NEWMA $t$ chart. It can also be noted that a small value of $\lambda$ in the VSI NEWMA $t$ chart is more sensitive to small shifts in a process. For example, when $\delta=0.1$ and $n=5$, the $\mathrm{ATS}_{1}=33.54$ of the VSI NEWMA $t$ chart with $\lambda=0.1$ performs better than the $\mathrm{ATS}_{1}=111.20$ of the chart with $\lambda=1.0$. In addition, with the increase in the value of $\delta$, the value of $\mathrm{ATS}_{1}$ decreases and converges to zero. Moreover, similar conclusions can be drawn for the VSI and FSI lower-sided NEWNA $t$ charts. For simplicity, these results are not presented here.

\section{A Simulated Example}

Similar to the example of Castagliola et al. [34], the data from a milk filling process are collected. The weight $X_{i}$ of each milk bottle is an interested quality characteristic. It is assumed that $X_{i}$ follows a normal distribution with mean $500 \mathrm{ml}$. While the variance of the normal distribution is unknown in practice. As a good alternative, $t$ type charts are used to monitor the process. According to the previous information of the filling process, a mean shift $\delta=-0.5$ needs to be detected quickly. For illustration, a dataset of 20 samples each with size $n=5$ are provided in the left side of Table 8 and the corresponding charting statistics of different charts are in the right side of the table. Since the shift size is small, a lower-sided VSI NEWMA $t$ chart with $\lambda=0.1$ is selected by practitioners. As a comparison, the 2-of-3 run rules $t$ chart and the traditional lower-sided EWMA $t$ chart are also presented. In addition, the VSI NEWMA $t$ chart with $\left(h_{S}, h_{L}\right)=(0.1,1.6)$ is also chosen for the process monitoring.

In Table 8 , the statistic $T_{i}$ is calculated according to equation (2) and the charting statistics $Y_{i}^{-}$of the traditional L-EWMA and L-NEWMA $t$ charts are calculated according to equations (4) and (7), respectively. Moreover, the lower control limits of $\mathrm{RR}_{2,3}-t$, L-EWMA $t$, and L-NEWMA $t$ charts are calculated as $-2.0762,-0.7695$, and -0.6811 , respectively, to maintain the desired $A R L_{0}=200$. The lower warning limit and the lower control limit of the lower-sided VSI NEWMA $t$ chart are calculated as -0.0426 and -0.6811 to maintain $\mathrm{ATS}_{0}=200$. All the values of these charting statistics are presented in Figure 1. It can be seen from this figure that, for the lower-sided $\mathrm{RR}_{2,3}-t$ control chart, two out of three sample points (samples 14 and 16) fall below the $\mathrm{LCL}^{-}=-2.0762$ and an out-of-control signal is given in the $16^{\text {th }}$ sample point, which means this chart takes 16 time units to detect the assignable cause. In addition, the traditional L-EWMA $t$ chart detects an out-of-control signal in the $14^{\text {th }}$ sample point. The L-NEWMA $t$ chart also gives an out-ofcontrol signal at the $14^{\text {th }}$ sample point, which means that both charts take 14 time units to detect the assignable cause. For the lower-sided VSI NEWMA $t$ chart, the charting statistic $Y_{i}^{-}$falls outside $\left[\mathrm{LWL}^{-}, 0\right]$ from the $5^{\text {th }}$ sample $\left(Y_{5}^{-}=0.2701>\mathrm{LWL}^{-}=0.0426\right)$, which causes a small sampling interval $h_{S}=0.1$ to get the subsequent samples. This fact leads to $\mathrm{ATS}_{1}=7.9$ time unit to detect the assignable cause. It can be seen that the proposed chart with VSI feature detects the shift in the process more quickly than the run rules, L-EWMA, and L-NEWMA charts. 
TABLE 6: The out-of-control ATS 1 profiles of the VSI and FSI upper-sided NEWMA $t$ charts when $\operatorname{ATS}_{0}=200$ and $n \in\{5,7\}$.

\begin{tabular}{|c|c|c|c|c|c|c|c|c|c|c|c|c|}
\hline \multirow{2}{*}{$n$} & \multirow{2}{*}{$\lambda$} & \multirow{2}{*}{ Chart } & \multirow{2}{*}{$\mathrm{UWL}^{+}$} & \multirow{2}{*}{$\mathrm{UCL}^{+}$} & \multicolumn{8}{|c|}{$\delta$} \\
\hline & & & & & 0.1 & 0.3 & 0.5 & 0.7 & 1.0 & 1.5 & 2 & 3 \\
\hline \multirow{16}{*}{5} & \multirow{2}{*}{0.1} & FSI & - & 0.6811 & 56.71 & 14.41 & 7.45 & 5.04 & 3.46 & 2.38 & 1.90 & 1.43 \\
\hline & & VSI & 0.0426 & 0.6811 & 33.54 & 3.97 & 1.44 & 0.76 & 0.40 & 0.24 & 0.19 & 0.14 \\
\hline & \multirow{2}{*}{0.2} & FSI & - & 1.1493 & 74.34 & 17.96 & 8.09 & 5.02 & 3.26 & 2.17 & 1.71 & 1.27 \\
\hline & & VSI & 0.0808 & 1.1493 & 49.17 & 5.24 & 1.56 & 0.76 & 0.38 & 0.22 & 0.17 & 0.13 \\
\hline & \multirow{2}{*}{0.3} & FSI & - & 1.5763 & 87.67 & 23.31 & 9.66 & 5.52 & 3.33 & 2.10 & 1.63 & 1.19 \\
\hline & & VSI & 0.1108 & 1.5763 & 62.91 & 7.49 & 1.85 & 0.81 & 0.38 & 0.21 & 0.16 & 0.13 \\
\hline & \multirow{2}{*}{0.4} & FSI & - & 1.9932 & 98.38 & 29.40 & 12.02 & 6.41 & 3.56 & 2.10 & 1.58 & 1.15 \\
\hline & & VSI & 0.1401 & 1.9932 & 74.42 & 10.85 & 2.36 & 0.92 & 0.40 & 0.21 & 0.16 & 0.12 \\
\hline & \multirow{2}{*}{0.5} & FSI & - & 2.4137 & 107.58 & 35.70 & 14.96 & 7.71 & 3.94 & 2.17 & 1.57 & 1.13 \\
\hline & & VSI & 0.1509 & 2.4137 & 82.86 & 14.36 & 3.12 & 1.09 & 0.44 & 0.22 & 0.16 & 0.11 \\
\hline & \multirow{2}{*}{0.75} & FSI & - & 3.4872 & 120.81 & 49.60 & 22.90 & 11.88 & 5.60 & 2.52 & 1.63 & 1.11 \\
\hline & & VSI & 0.2179 & 3.4872 & 101.44 & 25.44 & 6.58 & 2.06 & 0.64 & 0.25 & 0.16 & 0.11 \\
\hline & \multirow{2}{*}{0.9} & FSI & - & 4.1474 & 126.47 & 55.29 & 27.10 & 14.56 & 6.86 & 2.90 & 1.73 & 1.11 \\
\hline & & VSI & 0.2430 & 4.1474 & 108.09 & 31.61 & 9.31 & 3.03 & 0.85 & 0.29 & 0.17 & 0.11 \\
\hline & \multirow{2}{*}{1.0} & FSI & - & 4.5961 & 129.07 & 58.35 & 29.23 & 16.17 & 7.75 & 3.19 & 1.82 & 0.12 \\
\hline & & VSI & 0.2693 & 4.5961 & 111.20 & 35.64 & 11.53 & 3.95 & 1.05 & 0.32 & 0.18 & 0.11 \\
\hline \multirow{16}{*}{7} & \multirow{2}{*}{0.1} & FSI & - & 0.5958 & 43.21 & 10.51 & 5.64 & 3.90 & 2.75 & 1.94 & 1.58 & 1.13 \\
\hline & & VSI & 0.0419 & 0.5958 & 23.42 & 2.67 & 0.99 & 0.52 & 0.29 & 0.19 & 0.16 & 0.11 \\
\hline & \multirow{2}{*}{0.2} & FSI & - & 0.9854 & 54.50 & 11.74 & 5.58 & 3.63 & 2.47 & 1.72 & 1.36 & 1.04 \\
\hline & & VSI & 0.0770 & 0.9854 & 33.68 & 3.06 & 0.98 & 0.49 & 0.27 & 0.17 & 0.14 & 0.10 \\
\hline & \multirow{2}{*}{0.3} & FSI & - & 1.3314 & 66.24 & 14.12 & 5.98 & 3.66 & 2.38 & 1.61 & 1.26 & 1.01 \\
\hline & & VSI & 0.1040 & 1.3314 & 44.04 & 3.91 & 1.04 & 0.49 & 0.25 & 0.16 & 0.13 & 0.10 \\
\hline & \multirow{2}{*}{0.4} & FSI & - & 1.6610 & 76.68 & 17.23 & 6.79 & 3.87 & 2.36 & 1.54 & 1.20 & 1.01 \\
\hline & & VSI & 0.1298 & 1.6610 & 54.22 & 5.31 & 1.20 & 0.51 & 0.25 & 0.15 & 0.12 & 0.10 \\
\hline & \multirow{2}{*}{0.5} & FSI & - & 1.9866 & 84.97 & 21.03 & 7.94 & 4.25 & 2.42 & 1.50 & 1.16 & 1.00 \\
\hline & & VSI & 0.1552 & 1.9866 & 63.41 & 7.20 & 1.45 & 0.56 & 0.26 & 0.15 & 0.12 & 0.10 \\
\hline & \multirow{2}{*}{0.75} & FSI & - & 2.8192 & 101.69 & 31.24 & 12.04 & 5.88 & 2.84 & 1.50 & 1.13 & 1.00 \\
\hline & & VSI & 0.1982 & 2.8192 & 81.41 & 13.70 & 2.75 & 0.83 & 0.30 & 0.15 & 0.11 & 0.10 \\
\hline & \multirow{2}{*}{0.9} & FSI & - & 3.3416 & 109.32 & 37.10 & 15.24 & 7.34 & 3.30 & 1.55 & 1.13 & 1.00 \\
\hline & & VSI & 0.2350 & 3.3416 & 89.51 & 18.55 & 4.19 & 1.17 & 0.36 & 0.16 & 0.11 & 0.10 \\
\hline & \multirow{2}{*}{1.0} & FSI & - & 3.7043 & 112.28 & 40.68 & 17.30 & 8.44 & 3.70 & 1.61 & 1.13 & 1.00 \\
\hline & & VSI & 0.2605 & 3.7043 & 94.52 & 22.02 & 5.44 & 1.54 & 0.42 & 0.16 & 0.11 & 0.10 \\
\hline
\end{tabular}

TABLE 7: The out-of-control ATS 1 profiles of the VSI and FSI upper-sided NEWMA $t$ charts when $\operatorname{ATS}_{0}=200$ and $n \in\{9,11\}$.

\begin{tabular}{|c|c|c|c|c|c|c|c|c|c|c|c|c|}
\hline \multirow{2}{*}{$n$} & \multirow{2}{*}{$\lambda$} & \multirow{2}{*}{ Chart } & \multirow{2}{*}{$\mathrm{UWL}^{+}$} & \multirow{2}{*}{$\mathrm{UCL}^{+}$} & \multicolumn{8}{|c|}{$\delta$} \\
\hline & & & & & 0.1 & 0.3 & 0.5 & 0.7 & 1.0 & 1.5 & 2 & 3 \\
\hline \multirow{16}{*}{9} & \multirow{2}{*}{0.1} & FSI & - & 0.5614 & 35.53 & 8.61 & 4.74 & 3.33 & 2.38 & 1.73 & 1.36 & 1.02 \\
\hline & & VSI & 0.0395 & 0.5614 & 17.82 & 2.00 & 0.75 & 0.41 & 0.24 & 0.17 & 0.14 & 0.10 \\
\hline & \multirow{2}{*}{0.2} & FSI & - & 0.9227 & 44.74 & 9.12 & 4.50 & 3.01 & 2.11 & 1.73 & 1.16 & 1.02 \\
\hline & & VSI & 0.0721 & 0.9227 & 25.37 & 2.18 & 0.72 & 0.37 & 0.22 & 0.15 & 0.12 & 0.10 \\
\hline & \multirow{2}{*}{0.3} & FSI & - & 1.2367 & 54.12 & 10.42 & 4.60 & 2.93 & 1.98 & 1.36 & 1.08 & 1.00 \\
\hline & & VSI & 0.0966 & 1.2367 & 33.60 & 2.57 & 0.73 & 0.36 & 0.20 & 0.14 & 0.11 & 0.10 \\
\hline & \multirow{2}{*}{0.4} & FSI & - & 1.5315 & 63.18 & 12.25 & 4.94 & 2.97 & 1.91 & 1.28 & 1.05 & 1.00 \\
\hline & & VSI & 0.1197 & 1.5315 & 42.02 & 3.28 & 0.78 & 0.36 & 0.20 & 0.13 & 0.11 & 0.10 \\
\hline & \multirow{2}{*}{0.5} & FSI & - & 1.8214 & 70.77 & 14.61 & 5.50 & 3.08 & 1.89 & 1.24 & 1.04 & 1.00 \\
\hline & & VSI & 0.1423 & 1.8214 & 49.77 & 4.31 & 0.89 & 0.37 & 0.19 & 0.12 & 0.10 & 0.10 \\
\hline & \multirow{2}{*}{0.75} & FSI & - & 2.5577 & 87.52 & 22.06 & 7.90 & 3.83 & 1.98 & 1.19 & 1.02 & 1.00 \\
\hline & & VSI & 0.1998 & 2.5577 & 67.82 & 8.50 & 1.50 & 0.48 & 0.20 & 0.12 & 0.10 & 0.10 \\
\hline & \multirow{2}{*}{0.9} & FSI & - & 3.0280 & 96.41 & 27.38 & 9.96 & 4.64 & 2.16 & 1.20 & 1.02 & 1.00 \\
\hline & & VSI & 0.2366 & 3.0280 & 77.82 & 12.23 & 2.26 & 0.63 & 0.22 & 0.12 & 0.10 & 0.10 \\
\hline & \multirow{2}{*}{1.0} & FSI & - & 3.3526 & 100.79 & 30.40 & 11.56 & 5.31 & 2.33 & 1.20 & 1.02 & 1.00 \\
\hline & & VSI & 0.2619 & 3.3526 & 82.44 & 14.98 & 2.99 & 0.78 & 0.24 & 0.12 & 0.10 & 0.10 \\
\hline
\end{tabular}


TABle 7: Continued.

\begin{tabular}{|c|c|c|c|c|c|c|c|c|c|c|c|c|}
\hline \multirow{2}{*}{$n$} & \multirow{2}{*}{$\lambda$} & \multirow{2}{*}{ Chart } & \multirow{2}{*}{$\mathrm{UWL}^{+}$} & \multirow{2}{*}{$\mathrm{UCL}^{+}$} & \multicolumn{8}{|c|}{$\delta$} \\
\hline & & & & & 0.1 & 0.3 & 0.5 & 0.7 & 1.0 & 1.5 & 2 & 3 \\
\hline \multirow{16}{*}{11} & \multirow{2}{*}{0.1} & FSI & - & 0.5430 & 30.77 & 7.52 & 4.19 & 2.97 & 2.15 & 1.58 & 1.20 & 1.00 \\
\hline & & VSI & 0.0382 & 0.5430 & 14.45 & 1.62 & 0.61 & 0.34 & 0.22 & 0.16 & 0.12 & 0.10 \\
\hline & \multirow{2}{*}{0.2} & FSI & - & 0.8880 & 37.86 & 7.62 & 3.87 & 2.65 & 1.89 & 1.32 & 1.05 & 1.00 \\
\hline & & VSI & 0.0763 & 0.8880 & 20.72 & 1.73 & 0.58 & 0.31 & 0.19 & 0.13 & 0.11 & 0.10 \\
\hline & \multirow{2}{*}{0.3} & FSI & - & 1.1838 & 45.53 & 8.33 & 3.85 & 2.52 & 1.74 & 1.20 & 1.02 & 1.00 \\
\hline & & VSI & 0.1017 & 1.1838 & 27.37 & 1.95 & 0.57 & 0.29 & 0.18 & 0.12 & 0.10 & 0.10 \\
\hline & \multirow[b]{2}{*}{0.4} & FSI & - & 1.4634 & 54.05 & 9.56 & 4.01 & 2.49 & 1.66 & 1.14 & 1.01 & 1.00 \\
\hline & & VSI & 0.1258 & 1.4634 & 34.49 & 2.35 & 0.60 & 0.29 & 0.17 & 0.11 & 0.10 & 0.10 \\
\hline & \multirow{2}{*}{0.5} & FSI & - & 1.7343 & 61.15 & 11.14 & 4.31 & 2.52 & 1.61 & 1.11 & 1.01 & 1.00 \\
\hline & & VSI & 0.1490 & 1.7343 & 41.50 & 2.99 & 0.65 & 0.29 & 0.16 & 0.11 & 0.10 & 0.10 \\
\hline & \multirow{2}{*}{0.75} & FSI & - & 2.4219 & 77.92 & 16.78 & 5.75 & 2.88 & 1.60 & 1.07 & 1.00 & 1.00 \\
\hline & & VSI & 0.1892 & 2.4219 & 57.88 & 5.75 & 0.95 & 0.33 & 0.16 & 0.11 & 0.10 & 0.10 \\
\hline & \multirow{2}{*}{0.9} & FSI & - & 2.8575 & 86.06 & 21.02 & 7.14 & 3.33 & 1.66 & 1.07 & 1.00 & 1.00 \\
\hline & & VSI & 0.2232 & 2.8575 & 67.28 & 8.38 & 1.37 & 0.40 & 0.17 & 0.11 & 0.10 & 0.10 \\
\hline & \multirow{2}{*}{1.0} & FSI & - & 3.1667 & 91.00 & 24.05 & 8.34 & 3.76 & 1.73 & 1.07 & 1.00 & 1.00 \\
\hline & & VSI & 0.2474 & 3.1667 & 72.25 & 10.53 & 1.82 & 0.48 & 0.18 & 0.11 & 0.10 & 0.10 \\
\hline
\end{tabular}

TABLE 8: A set of sample data and the corresponding values of the charting statistics of different EWMA $t$ charts.

\begin{tabular}{lcccccccc}
\hline$i$ & $X_{i, 1}(\mathrm{ml})$ & $X_{i, 2}(\mathrm{ml})$ & $X_{i, 3}(\mathrm{ml})$ & $X_{i, 4}(\mathrm{ml})$ & $X_{i, 5}(\mathrm{ml})$ & $T_{i}$ & $Y_{i}^{-}(\mathrm{L}-\mathrm{EWMA})$ & $Y_{i}^{-}(\mathrm{L}-\mathrm{NEWMA})$ \\
\hline 1 & 499.51 & 499.55 & 500.80 & 499.82 & 499.86 & -0.3937 & -0.0394 & -0.0394 \\
2 & 500.50 & 500.29 & 502.29 & 499.11 & 499.91 & 0.8012 & 0.0000 & 0.0000 \\
3 & 502.37 & 499.45 & 499.95 & 498.84 & 499.00 & -0.1215 & -0.0122 & -0.0000 \\
4 & 499.24 & 499.67 & 501.11 & 500.18 & 498.81 & -0.4973 & -0.0607 & -0.0245 \\
5 & 498.95 & 499.91 & 497.79 & 499.57 & 499.27 & -2.4806 & -0.3027 & -0.2701 \\
6 & 502.17 & 497.83 & 500.23 & 499.74 & 500.11 & 0.0232 & -0.2701 & -0.1613 \\
7 & 500.75 & 501.32 & 500.20 & 501.76 & 497.86 & 0.5541 & -0.1877 & -0.2876 \\
8 & 500.18 & 499.72 & 499.06 & 499.94 & 499.77 & -1.4248 & -0.3114 & -0.3406 \\
9 & 500.71 & 497.56 & 499.04 & 498.81 & 501.18 & -0.8173 & -0.3620 & -0.6438 \\
10 & 498.89 & 499.51 & 498.62 & 499.21 & 499.92 & -3.3725 & -0.6630 & -0.5117 \\
11 & 499.47 & 501.70 & 500.04 & 500.21 & 499.87 & 0.6775 & -0.5290 & -0.6243 \\
12 & 498.50 & 500.01 & 498.81 & 500.39 & 499.39 & -1.6377 & -0.6398 & -0.5930 \\
13 & 499.71 & 499.32 & 498.70 & 501.31 & 500.27 & -0.3113 & -0.6070 & -0.8446 \\
14 & 498.13 & 498.59 & 499.73 & 499.60 & 499.22 & -3.1019 & -0.8572 & -0.7420 \\
15 & 499.83 & 500.70 & 500.37 & 499.05 & 500.31 & 0.1815 & -0.7533 & -1.0920 \\
16 & 499.90 & 498.38 & 498.39 & 498.28 & 498.07 & -4.2423 & -1.1022 & -1.4121 \\
17 & 499.73 & 499.40 & 499.08 & 498.76 & 498.60 & -4.2929 & -1.4213 & -2.8114 \\
18 & 498.63 & 499.43 & 499.43 & 499.31 & 498.94 & -5.4054 & -1.8197 & -2.2464 \\
19 & 498.99 & 498.34 & 498.58 & 499.40 & 499.04 & -6.0872 & -2.0854 \\
20 & 500.92 & 500.25 & 500.07 & 499.55 & 497.15 & -0.6361 & & -2.0787 \\
\hline
\end{tabular}

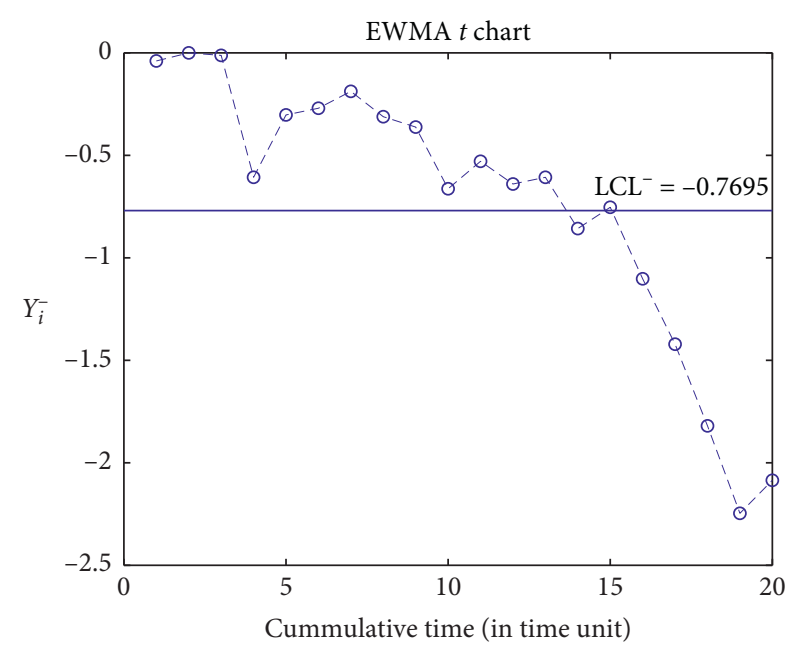

(a)

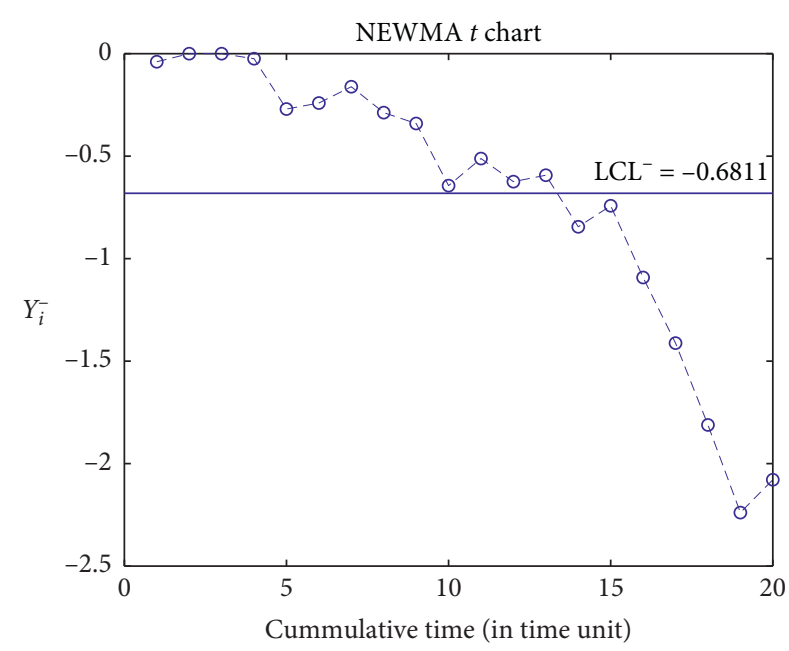

(b)

FIgure 1: Continued. 


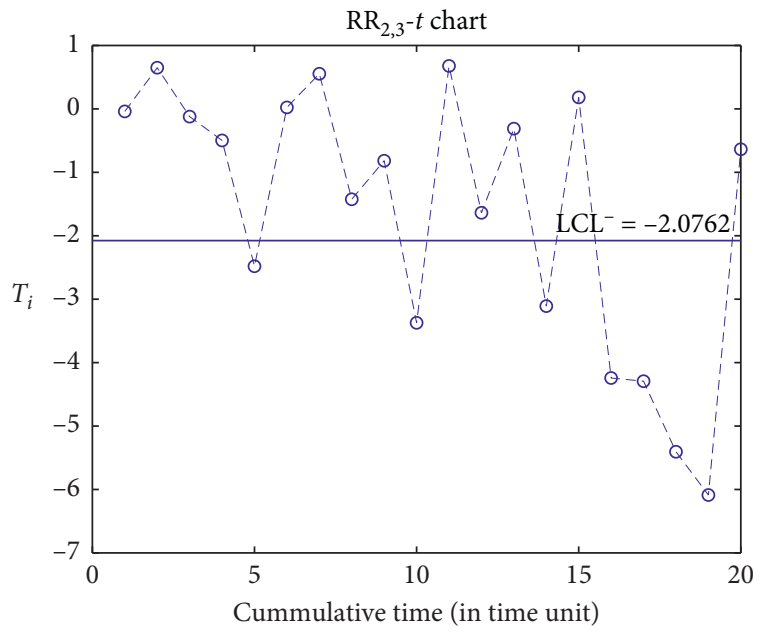

(c)

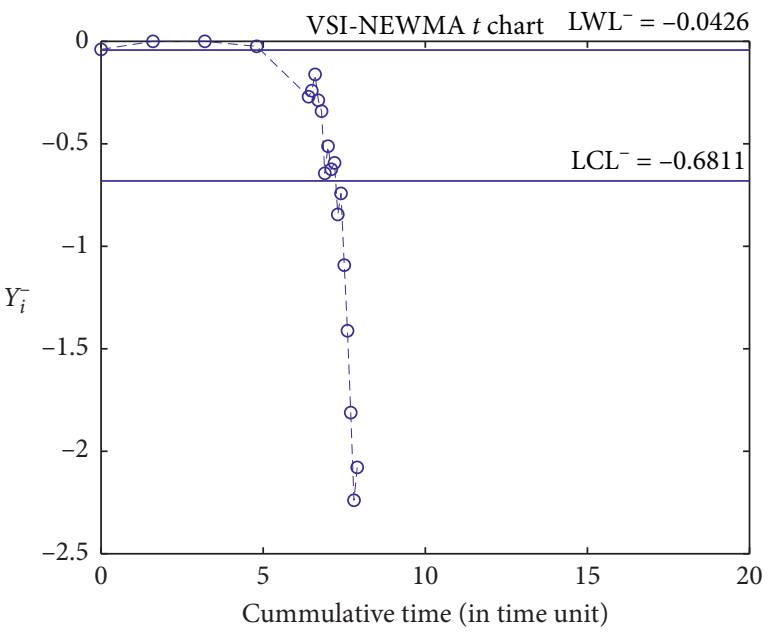

(d)

Figure 1: Different lower-sided $t$ charts applied to the dataset in Table 8. (a) The lower-sided EWMA $t$ chart, (b) the lower-sided NEWMA $t$ chart, (c) the lower-sided run rules $t$ chart, and (d) the lower-sided NEWMA $t$ chart with VSI.

\section{Conclusions and Recommendations}

In this paper, a new one-sided EWMA $t$ chart with a new resetting model is proposed for monitoring the process mean and the RL properties of the chart are obtained using a Monte Carlo method. Through numerous simulations, the results show that the ARL, MRL, and SDRL performances of the new one-sided EWMA $t$ chart are better than the ones of the traditional one-sided EWMA $t$ chart and several onesided run rules $t$ charts, especially for small values $\lambda$ and $\delta$. In addition, the performance of a new one-sided EWMA $t$ chart with VSI features is also studied. It is shown that the ATS performances of the VSI one-sided NEWMA $t$ chart are uniformly better than ones of the corresponding FSI chart.

The process parameters are assumed known in this paper. While in practice, they are often estimated from Phase I in-control samples. Therefore, the proposed chart needs to be further investigated when this assumption is violated. In addition, a fixed sample size is used in the proposed chart, while it can also be varied depending on the values of charting statistics. This is another interesting topic to be studied.

\section{Data Availability}

Some or all data, models, or code generated or used during the study are available from the corresponding author upon request.

\section{Conflicts of Interest}

The authors declare that they have no conflicts of interest.

\section{Acknowledgments}

This work was supported by the National Natural Science Foundation of China (nos. 71802110, 71872088, 11701437, and 71801049), Key Research Base of Philosophy and Social
Sciences in Jiangsu-Information Industry Integration Innovation and Emergency Management Research Center, Humanity and Social Science Youth Foundation of Ministry of Education of China (nos. 19YJA630061 and 17YJC630231), and Social Science Foundation of Jiangsu (no. 17GLB016).

\section{References}

[1] H. D. Maragah and W. H. Woodall, "The effect of autocorrelation on the retrospective X-chart," Journal of Statistical Computation and Simulation, vol. 40, no. 1-2, pp. 29-42, 1992.

[2] L. K. Chan and H. J. Cui, "Skewness correction $\bar{X}$ and R charts for skewed distributions," Naval Research Logistics, vol. 50, no. 6, pp. 555-573, 2003.

[3] G. Chen and S. W. Cheng, "Max chart: combining X-bar chart and S chart," Statistica Sinica, vol. 8, pp. 263-271, 1998.

[4] L. L. Ho and A. L. G. Trindade, "Economic design of an chart for short-run production," International Journal of Production Economics, vol. 120, no. 2, pp. 613-624, 2009.

[5] L. Zhang, G. Chen, and P. Castagliola, "On $t$ and EWMA $t$ charts for monitoring changes in the process mean," Quality and Reliability Engineering International, vol. 25, no. 8, pp. 933-945, 2009.

[6] K. P. Tran, "Designing of run rules $t$ control for monitoring changes in the process mean," Chemometrics and Intelligent Laboratory Systems, vol. 174, pp. 85-93, 2018.

[7] C. K. Sitt, M. B. C. Khoo, M. Shamsuzzaman, and C.-H. Chen, "The run sum $t$ control chart for monitoring process mean changes in manufacturing," The International Journal of Advanced Manufacturing Technology, vol. 70, no. 5-8, pp. 1487-1504, 2014.

[8] P. Castagliola, G. Celano, S. Fichera, and G. Nenes, "The variable sample size $t$ control chart for monitoring short production runs," International Journal of Advanced Manufacturing Technology, vol. 66, pp. 1353-1366, 2013.

[9] G. Celano, P. Castagliola, E. Trovato, and S. Fichera, "Shewhart and EWMA $t$ control charts for short production runs," Quality and Reliability Engineering International, vol. 27, no. 3, pp. 313-326, 2011. 
[10] G. Celano, P. Castagliola, and E. Trovato, "The economic performance of a CUSUMt control chart for monitoring short production runs," Quality Technology \& Quantitative Management, vol. 9, no. 4, pp. 329-354, 2012.

[11] G. Celano, P. Castagliola, S. Fichera, and G. Nenes, "Performance of $t$ control charts in short runs with unknown shift sizes," Computers \& Industrial Engineering, vol. 64, no. 1, pp. 56-68, 2013.

[12] M. E. Calzada and S. M. Scariano, "The synthetict and synthetic EWMAt charts," Quality Technology \& Quantitative Management, vol. 10, no. 1, pp. 37-56, 2013.

[13] Z. Chang and J. Sun, "AEWMA $t$ control chart for short production runs," Journal of Systems Science and Information, vol. 4, no. 5, pp. 444-459, 2017.

[14] R. B. Kazemzadeh, M. Karbasian, M. A. Babakhani, and M. A. Babakhani, "An EWMA $t$ chart with variable sampling intervals for monitoring the process mean," The International Journal of Advanced Manufacturing Technology, vol. 66, no. 1-4, pp. 125-139, 2013.

[15] P. Castagliola, G. Celano, and S. Psarakis, "Monitoring the coefficient of variation using EWMA charts," Journal of Quality Technology, vol. 43, no. 3, pp. 249-265, 2011.

[16] K. P. Tran, P. Castagliola, and G. Celano, "Monitoring the ratio of two normal variables using EWMA type control charts," Quality and Reliability Engineering International, vol. 32, no. 5, pp. 1853-1869, 2016.

[17] X. Hu, J. Zhong, S. Wu, and M. Qu, "Design of one-sided exponentially weighted moving average $t$ control chart," Journal of Applied Statistics and Management, vol. 39, pp. 112, 2020, in Chinese.

[18] J. Zhang, Z. Li, B. Chen, and Z. Wang, "A new exponentially weighted moving average control chart for monitoring the coefficient of variation," Computers \& Industrial Engineering, vol. 78, pp. 205-212, 2014.

[19] J. J. Divoky and R. W. Taylor, "Detecting process drift with combinations of trend and zonal supplementary runs rules," International Journal of Quality \& Reliability Management, vol. 12, no. 2, pp. 60-71, 1995.

[20] A. F. Bissell, "An attempt to unify the theory of quality control procedures," Journal of Applied Statistics, vol. 5, no. 2, pp. 113-128, 1978.

[21] S. W. Roberts, "Properties of control chart zone tests," Bell System Technical Journal, vol. 37, no. 1, pp. 83-114, 1958.

[22] C. W. Champ and W. H. Woodall, "Signal probabilities of runs rules supplementing a Shewhart control chart," Communication in Statistics-Simulation and Computation, vol. 26, no. $4,1997$.

[23] C. A. Lowry, C. W. Champ, and W. H. Woodall, "The performance of control charts for monitoring process variation," Communications in Statistics-Simulation and Computation, vol. 24, no. 2, pp. 409-437, 1995.

[24] A. C. Palm, "Tables of run length percentiles for determining the sensitivity of Shewhart control charts for averages with supplementary runs rules," Journal of Quality Technology, vol. 22, no. 4, pp. 289-298, 1990.

[25] G. Shmueli and A. Cohen, "Run-length distribution for control charts with runs and scans rules," Communications in Statistics-Theory and Methods, vol. 32, no. 2, pp. 475-495, 2003.

[26] Y. Zhang and P. Castagliola, "Run rules $\bar{X}$ charts when process parameters are unknown," International Journal of Reliability, Quality and Safety Engineering, vol. 17, no. 4, pp. 381-399, 2010.
[27] K. Tran, "The efficiency of the 4-out-of-5 runs rules scheme for monitoring the ratio of population means of a bivariate normal distribution," International Journal of Reliability, Quality and Safety Engineering, vol. 23, no. 5, pp. 1-26, 2016.

[28] A. Amdouni, P. Castagliola, H. Taleb, and G. Celano, "Onesided run rules control charts for monitoring the coefficient of variation in short production runs," European Journal of Industrial Engineering, vol. 10, no. 5, pp. 639-662, 2016.

[29] P. Castagliola, A. Achouri, H. Taleb, G. Celano, and S. Psarakis, "Monitoring the coefficient of variation using control charts with run rules," Quality Technology \& Quantitative Management, vol. 10, no. 1, pp. 75-94, 2013.

[30] M. R. Reynolds, R. W. Amin, J. C. Arnold, and J. A. Nachlas, "Charts with variable sampling intervals," Technometrics, vol. 30, no. 2, pp. 181-192, 1988.

[31] M. L. I. Coelho, M. A. Graham, and S. Chakraborti, "Nonparametric signed-rank control charts with variable sampling intervals," Quality and Reliability Engineering International, vol. 33, no. 8, pp. 2181-2192, 2017.

[32] A. Haq, "Weighted adaptive multivariate CUSUM charts with variable sampling intervals," Journal of Statistical Computation and Simulation, vol. 89, no. 3, pp. 1-14, 2018.

[33] H. D. Nguyen, K. P. Tran, and H. L. Heuchenne, "CUSUM control charts with variable sampling interval for monitoring the ratio of two normal variables," Quality and Reliability Engineering International, vol. 36, no. 2, pp. 474-497, 2020.

[34] P. Castagliola, P. E. Maravelakis, and F. O. Figueiredo, "The EWMA median chart with estimated parameters," IIE Transactions, vol. 48, no. 1, pp. 66-74, 2016. 